\title{
Racial and ethnic differences in predictors of vitamin D among pregnant women in south-eastern USA
}

\author{
Devika Chawla ${ }^{1}$, Julie L. Daniels ${ }^{1}$, Sara E. Benjamin-Neelon ${ }^{2}$, Bernard F. Fuemmeler ${ }^{3}$, Cathrine Hoyo ${ }^{4}$ and \\ Jessie P. Buckley ${ }^{5}$ \\ ${ }^{1}$ Department of Epidemiology, University of North Carolina Gillings School of Global Public Health, Chapel Hill, NC 27599-7400, USA \\ ${ }^{2}$ Department of Health, Behavior and Society, Johns Hopkins University Bloomberg School of Public Health, Baltimore, MD 21205, USA \\ ${ }^{3}$ Department of Health Behavior and Policy, Virginia Commonwealth University School of Medicine, Richmond, VA 23298, USA \\ ${ }^{4}$ Department of Biological Sciences, North Carolina State University, Raleigh, NC 27695, US A \\ ${ }^{5}$ Environmental Health and Engineering, Johns Hopkins University Bloomberg School of Public Health, Baltimore, MD 21205, USA
}

(Received 16 August 2018 - Final revision received 24 January 2019 - Accepted 30 January 2019)

Journal of Nutritional Science (2019), vol. 8, e8, page 1 of 6

doi:10.1017/jns.2019.4

Abstract

Insufficient vitamin D during pregnancy increases risk of adverse outcomes, with known differences by race/ethnicity. We sought to determine whether predictors of vitamin D insufficiency vary by race/ethnicity in an ethnically diverse pregnancy cohort. Plasma 25-hydroxyvitamin D concentrations and patient characteristics were measured at first prenatal visit to prenatal clinics in south-eastern USA between 2009 and 2011 ( $n$ 504). Prevalence ratios (PR) and $95 \%$ CI were estimated using multivariable regression to quantify predictors of vitamin D insufficiency, overall and by race/ethnicity. In race/ethnicity-stratified models, season was most associated with vitamin D insufficiency among non-Hispanic white women; PR for winter $v$. summer were $3.58(95 \%$ CI 1.64, 7.81) for non-Hispanic white, 1.52 (95\% CI 1.18, 1.95) for Hispanic and 1.14 (95\% CI 0.99, 1.30) for non-Hispanic black women. Although women with darker skin tones are most vulnerable to prenatal vitamin D insufficiency, season may be more strongly associated with insufficiency among women with lighter skin tones.

Key words: Vitamin D: 25-Hydroxyvitamin D: Race: Ethnicity

Over half of pregnant women in the USA have suboptimal vitamin D levels, and non-Hispanic black women are disproportionally affected ${ }^{(1,2)}$. Recent epidemiological studies suggest that insufficient prenatal vitamin D increases risk of adverse pregnancy outcomes, including pre-eclampsia, pregnancy loss, preterm delivery and fetal growth restriction ${ }^{(3-5)}$. However, pregnancy-specific guidelines have been widely accepted and current guidelines for vitamin D sufficiency in the general population differ among authoritative bodies ${ }^{(6)}$.

Race and ethnicity are known to be strong predictors of vitamin $\mathrm{D}$, as measured by 25 -hydroxyvitamin $\mathrm{D}(25(\mathrm{OH}) \mathrm{D})$ concentrations. Previous studies measuring 25(OH)D concentrations during pregnancy have reported higher concentrations in non-Hispanic white women compared with Hispanic and non-Hispanic black women ${ }^{(7,8)}$. Some studies suggest that the aetiological effects of vitamin $\mathrm{D}$ on health outcomes differ by race/ethnicity ${ }^{(4,5,7)}$. Moreover, a recent study reported that effects of prenatal $25(\mathrm{OH}) \mathrm{D}$ concentrations on birth outcomes were modified by race/ethnicity ${ }^{(9)}$.

Despite these known differences, few studies have assessed whether predictors of $25(\mathrm{OH}) \mathrm{D}$ concentrations also vary by race/ethnicity. Understanding the relative importance of

Abbreviation: 25(OH)D, 25-hydroxyvitamin D.

* Corresponding author: Jessie P. Buckley, email Jessie.Buckley@jhu.edu

(C) The Author(s) 2019. This is an Open Access article, distributed under the terms of the Creative Commons Attribution licence (http://creativecommons.org/licenses/by/4.0/), which permits unrestricted re-use, distribution, and reproduction in any medium, provided the original work is properly cited. 
vitamin D predictors in different race/ethnicity groups is important for (1) identifying populations at high risk of vitamin D deficiency during pregnancy, (2) identifying race/ ethnicity-specific modifiable risk factors for vitamin D deficiency and (3) adequately modelling effect measure modification by race/ethnicity in aetiological studies of vitamin D.

To address this gap, we measured plasma concentrations of $25(\mathrm{OH}) \mathrm{D}$, a biomarker of vitamin D exposure, in an ethnically diverse pregnancy cohort in south-eastern USA and examined how predictors of $25(\mathrm{OH}) \mathrm{D}$ concentrations vary by race/ ethnicity.

\section{Methods}

\section{Study population}

The Newborn Epigenetic Study (NEST) followed a cohort of pregnant women who were seeking prenatal care from four healthcare facilities in a south-eastern US county from 2009 to 2011. Women were eligible to participate if they were 18 years of age or older, spoke English or Spanish, and planned to receive obstetric care at one of two facilities in Durham county (latitude: $36^{\circ} \mathrm{N}$ ). Interviewers administered questionnaires to women at enrolment to ascertain information on sociodemographic characteristics and behaviours. Additional details on study design of the NEST are available elsewhere ${ }^{(10)}$. Plasma 25(OH)D concentrations were measured in the first 510 enrolled pregnant women, of whom 504 had valid measures and were included in this analysis. The NEST was approved by the Duke University Institutional Review Board and written consent was obtained from all women participating as study subjects.

\section{Vitamin D measurement}

Participating mothers provided a non-fasting blood sample at the time of enrolment, typically during the 1 st or 2 nd trimester (median 11.3 (interquartile range 8.9-15.6) weeks of gestation). Plasma samples were aliquoted and stored at $-80^{\circ} \mathrm{C}$ until analysis. We measured plasma concentrations of 25 $(\mathrm{OH}) \mathrm{D}$ using the commercially available immunodiagnostic system (IDS Fountain Hills AZ) enzyme immunoassay (catalogue no. AC57F1) (Craft Technologies, Inc.). We performed the immunoassay in duplicate using $25 \mu \mathrm{l}$ plasma, according to the manufacturer's instructions, and \% CV of all sample duplicates was $<13 \%$. We categorised women as having sufficient $(\geq 50 \mathrm{nmol} / \mathrm{l})$ or insufficient $(<50 \mathrm{nmol} / \mathrm{l})$ vitamin $\mathrm{D}$ status (hereafter referred to as vitamin D sufficient or insufficient), according to the Institute of Medicine (now the National Academy of Medicine) guidelines ${ }^{(11,12)}$.

\section{Patient characteristics}

We identified potential predictors of vitamin $\mathrm{D}$ from current literature. We assigned season of blood draw based on the date of the sample collection and categorised the season as winter (December-February), spring (March-May), summer (JuneAugust) and autumn (September-November). We ascertained self-reported maternal characteristics, including pre-pregnancy weight, height, age, education, marital status, parity, prenatal vitamin use, multivitamin use and smoking, by questionnaire at enrolment. Women self-identified as non-Hispanic white, non-Hispanic black, or Hispanic. We calculated pre-pregnancy BMI in $\mathrm{kg} / \mathrm{m}^{2}$ as self-reported pre-pregnancy weight/ (height $\mathrm{x}$ height) and categorised women as underweight $(<18.5 \mathrm{~kg} /$ $\mathrm{m}^{2}$ ), normal weight $\left(18.5-24.9 \mathrm{~kg} / \mathrm{m}^{2}\right)$, overweight $(25 \cdot 0$ $\left.29.9 \mathrm{~kg} / \mathrm{m}^{2}\right)$ or obese $\left(\geq 30 \cdot 0 \mathrm{~kg} / \mathrm{m}^{2}\right)$. We defined maternal smoking as any current smoking at time of enrolment. Similarly, we defined prenatal vitamin and (non-prenatal) multivitamins as any current use at time of enrolment. Gestational age at enrolment was derived from last menstrual period and categorised as 1 st ( $<14$ completed weeks of gestation) or 2 nd trimester (14-27 completed weeks of gestation).

\section{Statistical analysis}

We tabulated covariate distributions by vitamin D status (defined as insufficient or sufficient) and computed mean 25 $(\mathrm{OH}) \mathrm{D}$ concentrations by covariate groupings, overall and by race/ethnicity. $25(\mathrm{OH}) \mathrm{D}$ concentrations were normally distributed in our population. To evaluate independent predictors of low $25(\mathrm{OH}) \mathrm{D}$ concentrations in our study population, we estimated prevalence ratios and $95 \%$ CI by simultaneously including all patient characteristics (defined above) in log-binomial regression models predicting prevalence of insufficient vitamin $\mathrm{D}$ status ${ }^{(13)}$. We also ran models stratified by race/ethnicity to examine predictors separately for non-Hispanic white, non-Hispanic black and Hispanic women (Table 3). To formally test whether predictors of $25(\mathrm{OH}) \mathrm{D}$ concentrations differed by race/ethnicity, we fit an equivalent model that included product terms between race/ethnicity and all predictors ${ }^{(14)}$. For main effects, we considered estimates to be statistically significant at $P<0 \cdot 05$. We considered there to be significant differences by race/ethnicity if the Wald type I $P$ value for the product term was $<0 \cdot 1$. In sensitivity analyses, we ran linear regression models examining predictors of continuous plasma $25(\mathrm{OH}) \mathrm{D}$ concentrations and found similar results (data not shown). We performed statistical analyses in SAS 9.3 (SAS Institute).

\section{Ethical standards disclosure}

This study was conducted according to the guidelines laid down in the Declaration of Helsinki and all procedures involving human subjects/patients were approved by the Institutional Review Board of Duke University Medical Center. Written informed consent was obtained from all patients.

\section{Results and discussion}

We report the distribution of participant characteristics in the overall population and among women with sufficient or insufficient vitamin D status in Table 1 and observed mean 25(OH) D concentrations, overall and by race/ethnicity, in Table 2 . The study population was distributed across non-Hispanic white (n 144), non-Hispanic black (n 191) and Hispanic (n 169) women. The prevalence of vitamin D insufficiency 
Table 1. Study population characteristics for women with overall, sufficient and insufficient vitamin D status*

(Numbers of participants and percentages)

\begin{tabular}{|c|c|c|c|c|c|c|}
\hline & \multicolumn{2}{|c|}{$\begin{array}{l}\text { Overall } \\
(n 504)\end{array}$} & \multicolumn{2}{|c|}{$\begin{array}{c}\text { Sufficient } \\
(n 135)\end{array}$} & \multicolumn{2}{|c|}{$\begin{array}{c}\text { Insufficient } \\
(n \text { 369) }\end{array}$} \\
\hline & $n$ & $\%$ & $n$ & $\%$ & $n$ & $\%$ \\
\hline \multicolumn{7}{|l|}{ Season of blood draw } \\
\hline Winter & 113 & 23 & 14 & 10 & 99 & 27 \\
\hline Spring & 157 & 31 & 28 & 21 & 129 & 35 \\
\hline Summer & 140 & 28 & 58 & 43 & 82 & 22 \\
\hline Autumn & 94 & 19 & 35 & 26 & 59 & 16 \\
\hline \multicolumn{7}{|l|}{ Race/ethnicity } \\
\hline Non-Hispanic white & 144 & 29 & 76 & 56 & 68 & 18 \\
\hline Non-Hispanic black & 191 & 38 & 17 & 13 & 174 & 47 \\
\hline Hispanic & 169 & 34 & 42 & 31 & 127 & 34 \\
\hline \multicolumn{7}{|l|}{ Pre-pregnancy BMI } \\
\hline Underweight & 17 & 3 & 7 & 5 & 10 & 3 \\
\hline Normal & 191 & 38 & 70 & 52 & 121 & 33 \\
\hline Overweight & 153 & 30 & 35 & 26 & 118 & 32 \\
\hline Obese & 143 & 29 & 23 & 17 & 120 & 32 \\
\hline \multicolumn{7}{|l|}{ Maternal age at delivery (years) } \\
\hline$<25$ & 172 & 34 & 29 & 21 & 143 & 39 \\
\hline $25-29$ & 137 & 27 & 33 & 24 & 104 & 28 \\
\hline $30-34$ & 121 & 24 & 49 & 36 & 72 & 20 \\
\hline$\geq 35$ & 74 & 15 & 24 & 18 & 50 & 14 \\
\hline \multicolumn{7}{|l|}{ Maternal education } \\
\hline$<$ High school graduate & 169 & 33 & 29 & 21 & 140 & 38 \\
\hline High school graduate/GED & 109 & 22 & 26 & 19 & 83 & 22 \\
\hline Some college & 76 & 15 & 11 & 8 & 65 & 18 \\
\hline College graduate & 147 & 29 & 67 & 50 & 80 & 22 \\
\hline Missing & 3 & & 2 & & 1 & \\
\hline \multicolumn{7}{|l|}{ Marital status } \\
\hline Never married & 141 & 28 & 17 & 13 & 124 & 34 \\
\hline Married & 191 & 38 & 81 & 60 & 110 & 30 \\
\hline Living with partner & 138 & 28 & 32 & 24 & 106 & 29 \\
\hline Divorced/widowed/other & 28 & 6 & 4 & 3 & 24 & 7 \\
\hline Missing & 6 & & 1 & & 5 & \\
\hline \multicolumn{7}{|l|}{ Parity } \\
\hline 0 & 176 & 35 & 45 & 33 & 131 & 36 \\
\hline $1+$ & 328 & 65 & 90 & 67 & 238 & 65 \\
\hline \multicolumn{7}{|l|}{ Prenatal vitamin } \\
\hline Yes & 279 & 56 & 82 & 62 & 198 & 55 \\
\hline No & 216 & 44 & 51 & 38 & 165 & 45 \\
\hline Missing & 8 & & 2 & & 6 & \\
\hline \multicolumn{7}{|l|}{ Additional multivitamins } \\
\hline Yes & 17 & 3 & 9 & 7 & 8 & 2 \\
\hline No & 474 & 97 & 122 & 93 & 352 & 98 \\
\hline Missing & 13 & & 4 & & 9 & \\
\hline \multicolumn{7}{|l|}{ Smoking during pregnancy } \\
\hline Yes & 79 & 16 & 16 & 12 & 62 & 17 \\
\hline No & 413 & 84 & 116 & 88 & 299 & 83 \\
\hline Missing & 11 & & 3 & & 8 & \\
\hline \multicolumn{7}{|l|}{ Gestational age at enrolment } \\
\hline 1st trimester & 312 & 62 & 90 & 67 & 222 & 60 \\
\hline 2nd trimester & 192 & 38 & 45 & 33 & 147 & 40 \\
\hline
\end{tabular}

GED, general education development.

* Insufficient vitamin D status is defined as plasma concentrations $<50 \mathrm{nmol} / \mathrm{l}$, while sufficient vitamin D status is defined as plasma concentrations $\geq 50 \mathrm{nmol} / \mathrm{l}$.

was $47 \%$ for non-Hispanic white women, $91 \%$ for non-Hispanic black women and $75 \%$ for Hispanic women. Non-Hispanic black women in winter months had the lowest observed $25(\mathrm{OH}) \mathrm{D}$ concentrations of any group (mean $=28.8$ $(\mathrm{SD}=9.4) \mathrm{nmol} / \mathrm{l})$, while white women in summer months had the highest $($ mean $=58 \cdot 3(\mathrm{sD}=11 \cdot 8) \mathrm{nmol} / \mathrm{l})$. Mean concentrations of $25(\mathrm{OH}) \mathrm{D}$ were lower among obese compared with normal-weight women among all race/ethnicity groups. Women who used prenatal vitamins had similar observed 25 $(\mathrm{OH}) \mathrm{D}$ concentrations to those who did not (Table 2).
In adjusted models, season of blood draw (specifically winter) and race/ethnicity (all race/ethnicity groups) were the strongest predictors of vitamin D insufficiency in the overall study population (Table 3). Season of blood draw remained the strongest predictor in race-specific adjusted models, and was an especially strong predictor of vitamin D insufficiency for non-Hispanic white women. Higher pre-pregnancy BMI was significantly associated with higher prevalence of vitamin $\mathrm{D}$ insufficiency in the overall model, and in stratified models persisted as a significant predictor for non-Hispanic black women (Table 3). Lack of prenatal vitamin use was not a statistically significant predictor of vitamin $\mathrm{D}$ insufficiency in any racial/ethnic groups (Table 3). Lower maternal education was significantly associated with higher prevalence of vitamin D insufficiency in the overall models, and was especially strong for non-Hispanic white and Hispanic women (Table 3).

In models stratified by race/ethnicity, we observed some statistically significant differences in predictors of vitamin D insufficiency. Relationships of season and marital status with vitamin D insufficiency were significantly different among white women as compared with non-Hispanic black $(P<0 \cdot 01$ for season, $P=0.05$ for marital status) or Hispanic women ( $P<0.01$ for season, $P=0.05$ for marital status). In addition, the relationship between parity and vitamin $\mathrm{D}$ insufficiency was significantly different among non-Hispanic white women as compared with among non-Hispanic black women $(P=$ $0 \cdot 02)$, but not as compared with Hispanic women $(P=0 \cdot 18)$.

We identified several predictors of vitamin $\mathrm{D}$ insufficiency in pregnant women that differed according to race/ethnicity, including season of blood draw, parity and marital status. While season and race/ethnicity are known to be independently associated with vitamin D status, our study is the first to quantify differences in seasonal patterns of vitamin $\mathrm{D}$ insufficiency in some but not all race/ethnic groups in a multiethnic cohort of pregnant women.

Our results are consistent with findings from a recent study of three cohorts in the USA showing that predicted seasonal variations in prenatal $25(\mathrm{OH}) \mathrm{D}$ concentrations were stronger for white women as compared with black women ${ }^{(8)}$, but additionally compares seasonal patterns among Hispanic women. In our sample, seasonal variation for Hispanic women was less pronounced than among non-Hispanic white women but slightly stronger than among non-Hispanic black women. These differences in seasonal patterns persisted after adjusting for socio-economic status and other important predictors, suggesting that differences may be biologically driven. Darker skin tones require more UVB sunlight to generate previtamin $\mathrm{D}$, which subsequently results in $25(\mathrm{OH}) \mathrm{D}$ in the body. This may, in part, explain why non-Hispanic black women have higher rates of vitamin $\mathrm{D}$ insufficiency than other racial/ethnic groups. Moreover, women with darker skin tones may be less sensitive to seasonal variations in sun exposure, since melatonin in darker skin tones reduces the efficiency of UVB absorption $^{(15)}$. This may result in more dramatic seasonal patterns of $25(\mathrm{OH}) \mathrm{D}$ (e.g. higher peaks, lower nadirs) among non-Hispanic white women as compared with black and Hispanic women, which we observed in our study. Although we lacked information on skin tone, country of origin and 
Table 2. Plasma 25-hydroxyvitamin $\mathrm{D}$ concentrations (nmol/l) in the study population, overall and by race/ethnicity (Mean values and standard deviations)

\begin{tabular}{|c|c|c|c|c|c|c|c|c|}
\hline & \multicolumn{2}{|c|}{ Overall ( $n$ 504) } & \multicolumn{2}{|c|}{ White ( $n$ 144) } & \multicolumn{2}{|c|}{ Black ( $n$ 191) } & \multicolumn{2}{|c|}{ Hispanic ( $n$ 169) } \\
\hline & Mean & SD & Mean & SD & Mean & SD & Mean & SD \\
\hline Overall & $41 \cdot 3$ & $14 \cdot 3$ & $51 \cdot 1$ & $12 \cdot 9$ & 33.4 & 11.8 & 41.8 & $12 \cdot 6$ \\
\hline \multicolumn{9}{|l|}{ Season } \\
\hline Winter & 35.0 & $13 \cdot 1$ & 45.5 & 13.1 & 28.8 & 9.4 & 33.7 & 11.4 \\
\hline Spring & 38.5 & $12 \cdot 8$ & $46 \cdot 7$ & $11 \cdot 2$ & 31.7 & $10 \cdot 6$ & 39.4 & $12 \cdot 6$ \\
\hline Summer & 47.8 & 13.5 & 58.3 & 11.8 & 39.0 & 11.2 & 48.0 & 11.1 \\
\hline Autumn & 43.8 & 14.7 & 53.3 & $11 \cdot 8$ & 35.2 & $14 \cdot 2$ & 43.4 & 9.8 \\
\hline \multicolumn{9}{|l|}{ Pre-pregnancy BMI } \\
\hline Underweight & $42 \cdot 9$ & $13 \cdot 8$ & 49.3 & 11.5 & 33.0 & $16 \cdot 3$ & 43.7 & $10 \cdot 0$ \\
\hline Normal & $45 \cdot 0$ & 14.6 & $52 \cdot 7$ & 13.5 & 37.3 & $13 \cdot 15$ & 43.4 & 12.4 \\
\hline Overweight & $40 \cdot 1$ & $14 \cdot 3$ & 51.6 & 13.6 & $30 \cdot 8$ & 9.8 & 41.6 & $13 \cdot 1$ \\
\hline Obese & 37.3 & $12 \cdot 8$ & 47.4 & $10 \cdot 9$ & 31.9 & $11 \cdot 0$ & $39 \cdot 3$ & $12 \cdot 1$ \\
\hline \multicolumn{9}{|l|}{ Maternal age (years) } \\
\hline$<25$ & 38.4 & $13 \cdot 2$ & 50.7 & 14.6 & 33.8 & 11.9 & 41.4 & $11 \cdot 2$ \\
\hline $25-29$ & $40 \cdot 0$ & $15 \cdot 2$ & $50 \cdot 7$ & $14 \cdot 0$ & $31 \cdot 6$ & 13.0 & $40 \cdot 1$ & $12 \cdot 8$ \\
\hline $30-34$ & $45 \cdot 6$ & 14.4 & 53.0 & $11 \cdot 8$ & 33.2 & 9.0 & 43.4 & 14.4 \\
\hline$\geq 35$ & 43.3 & $13 \cdot 1$ & $48 \cdot 2$ & $12 \cdot 4$ & $35 \cdot 8$ & $12 \cdot 2$ & 43.9 & $12 \cdot 1$ \\
\hline \multicolumn{9}{|l|}{ Maternal education } \\
\hline$<$ High school graduate & 39.0 & $12 \cdot 9$ & 43.8 & $13 \cdot 0$ & 33.9 & $12 \cdot 7$ & 41.3 & $12 \cdot 2$ \\
\hline High school graduate/GED & 39.5 & $15 \cdot 2$ & 57.4 & $12 \cdot 7$ & 33.9 & $12 \cdot 6$ & $40 \cdot 3$ & $13 \cdot 3$ \\
\hline Some college & $36 \cdot 1$ & $12 \cdot 2$ & 44.7 & $11 \cdot 8$ & $32 \cdot 1$ & $10 \cdot 8$ & 41.4 & $10 \cdot 5$ \\
\hline College graduate & $47 \cdot 7$ & $14 \cdot 2$ & 51.9 & $12 \cdot 4$ & 33.0 & $10 \cdot 6$ & 50.5 & $12 \cdot 1$ \\
\hline \multicolumn{9}{|l|}{ Marital status } \\
\hline Never married & $36 \cdot 3$ & 12.5 & 53.5 & 13.0 & 33.9 & 11.9 & $38 \cdot 7$ & 9.7 \\
\hline Married & $46 \cdot 6$ & $14 \cdot 2$ & $51 \cdot 3$ & $12 \cdot 6$ & 32.4 & $10 \cdot 1$ & $43 \cdot 8$ & $13 \cdot 7$ \\
\hline Living with partner & $40 \cdot 2$ & $14 \cdot 1$ & 50.6 & $15 \cdot 6$ & $32 \cdot 6$ & $12 \cdot 4$ & $42 \cdot 3$ & $12 \cdot 7$ \\
\hline Divorced/widowed/other & 37.2 & $12 \cdot 8$ & 41.4 & $13 \cdot 2$ & 34.4 & 13.4 & 38.5 & 12.5 \\
\hline \multicolumn{9}{|l|}{ Parity } \\
\hline 0 & $42 \cdot 1$ & $13 \cdot 2$ & 50.1 & 11.9 & $35 \cdot 3$ & $12 \cdot 2$ & 41.3 & 9.4 \\
\hline $1+$ & $40 \cdot 8$ & 14.9 & 51.9 & 13.7 & $32 \cdot 3$ & 11.5 & $42 \cdot 0$ & 13.5 \\
\hline \multicolumn{9}{|l|}{ Prenatal vitamin } \\
\hline Yes & 43.0 & $14 \cdot 2$ & $51 \cdot 1$ & $13 \cdot 0$ & $34 \cdot 2$ & $12 \cdot 3$ & $43 \cdot 6$ & $12 \cdot 3$ \\
\hline No & 39.0 & $14 \cdot 3$ & $51 \cdot 0$ & $13 \cdot 0$ & $32 \cdot 3$ & $11 \cdot 3$ & $39 \cdot 3$ & 12.9 \\
\hline Missing & $45 \cdot 1$ & 7.5 & $n<5$ & & $n<5$ & & $n<5$ & \\
\hline \multicolumn{9}{|l|}{ Additional multivitamin } \\
\hline Yes & 47.8 & $16 \cdot 3$ & 60.5 & 8.6 & 35.4 & 14.0 & 52.4 & 3.3 \\
\hline No & $41 \cdot 0$ & $14 \cdot 2$ & 50.6 & 13.0 & 33.1 & $11 \cdot 6$ & 41.5 & $12 \cdot 6$ \\
\hline Missing & 43.9 & $13 \cdot 0$ & $n<5$ & & $39 \cdot 0$ & 14.4 & $48 \cdot 0$ & 9.2 \\
\hline \multicolumn{9}{|l|}{ Smoking during pregnancy } \\
\hline No & $42 \cdot 1$ & 14.0 & 51.4 & $13 \cdot 1$ & 33.8 & 11.4 & 41.8 & $12 \cdot 1$ \\
\hline Yes & 37.0 & $15 \cdot 2$ & 48.8 & $12 \cdot 2$ & 31.9 & $12 \cdot 9$ & 44.7 & 20.5 \\
\hline Missing & 39.4 & 14.5 & $n<5$ & & 37.8 & $12 \cdot 2$ & 38.4 & $18 \cdot 1$ \\
\hline \multicolumn{9}{|c|}{ Gestational age at enrolment (weeks) } \\
\hline $0-10$ & 41.6 & 14.9 & 51.5 & $14 \cdot 2$ & 31.9 & 11.5 & 42.5 & 11.6 \\
\hline $11-20$ & $41 \cdot 0$ & $13 \cdot 7$ & $50 \cdot 7$ & 11.9 & 33.3 & $10 \cdot 7$ & 41.0 & $12 \cdot 7$ \\
\hline $21-35$ & 41.7 & $15 \cdot 3$ & 51.6 & $15 \cdot 5$ & 37.0 & $15 \cdot 3$ & $43 \cdot 7$ & 14.0 \\
\hline
\end{tabular}

GED, general education development.

detailed Hispanic ethnicity, our finding suggests that seasonal patterns of prenatal vitamin $\mathrm{D}$ differ by ethnicity as well as by race.

While associations between insufficient vitamin D status and parity and marital status differed significantly across race/ethnicity, these variables were not significant predictors of vitamin D insufficiency in any group and sample size was limited in stratified models. Being married was associated with slightly lower prevalence of insufficient vitamin D status for Hispanic women, but not for non-Hispanic black or non-Hispanic white women. This is probably explained by unmeasured confounding from broader socio-economic factors that are associated with marital status and may differ by race/ethnicity. Broadly, these results suggest that some - but not all - predictors of prenatal vitamin D may differ by race/ethnicity.
In overall models, season and race/ethnicity were strong predictors of vitamin $\mathrm{D}$ insufficiency during pregnancy as has been observed in other studies ${ }^{(8)}$. Pre-pregnancy BMI was a significant predictor of prenatal vitamin $\mathrm{D}$ insufficiency in the adjusted model, with overweight and obese women having an increased prevalence of vitamin $\mathrm{D}$ insufficiency. These results contribute to the small but growing literature that increased BMI may be associated with lower $25(\mathrm{OH}) \mathrm{D}$ concentrations $^{(16)}$. Prenatal vitamin use and multivitamin use were not significant predictors of insufficiency. Most prenatal vitamins include vitamin $\mathrm{D}$, but dosage varies by brand and adequacy of the dosing for vitamin $\mathrm{D}$ supplementation has been called into question ${ }^{(17)}$.

Understanding racial/ethnic differences in overall risk and predictors of vitamin $\mathrm{D}$ insufficiency has clinical implications 
Table 3. Predictors of insufficient vitamin D status, overall and by race/ethnicity* (Prevalence ratios (PR) and $95 \%$ confidence intervals)

\begin{tabular}{|c|c|c|c|c|c|c|c|c|}
\hline & \multicolumn{2}{|c|}{ Overall } & \multicolumn{2}{|c|}{ White } & \multicolumn{2}{|c|}{ Black $†$} & \multicolumn{2}{|c|}{ Hispanicł } \\
\hline & PR & $95 \% \mathrm{Cl}$ & PR & $95 \% \mathrm{Cl}$ & PR & $95 \% \mathrm{Cl}$ & PR & $95 \% \mathrm{Cl}$ \\
\hline Season & & & & & $P<0.01 \S$ & & $P<0.01 \S$ & \\
\hline Winter & 1.55 & $1 \cdot 32,1 \cdot 81$ & 3.58 & $1.64,7.81$ & $1 \cdot 14$ & $0.99,1.30$ & 1.52 & $1.18,1.95$ \\
\hline Spring & 1.40 & $1.20,1.63$ & 3.56 & $1.63,7.74$ & $1 \cdot 16$ & $1.01,1.32$ & 1.28 & $1.00,1.62$ \\
\hline Summer & Ref & & Ref & & Ref & & Ref & \\
\hline Autumn & $1 \cdot 14$ & $0.94,1.40$ & 1.72 & $0.69,4.29$ & 1.00 & $0.83,1.20$ & 1.36 & $1.02,1.83$ \\
\hline \multicolumn{9}{|l|}{ Race/ethnicity } \\
\hline White & Ref & & $\mathrm{N} / \mathrm{A}$ & & $\mathrm{N} / \mathrm{A}$ & & N/A & \\
\hline Black & 1.56 & $1.28,1.90$ & $\mathrm{~N} / \mathrm{A}$ & & $\mathrm{N} / \mathrm{A}$ & & $\mathrm{N} / \mathrm{A}$ & \\
\hline Hispanic & 1.34 & $1.07,1.69$ & $N / A$ & & $\mathrm{~N} / \mathrm{A}$ & & N/A & \\
\hline Pre-pregnancy BMI & & & & & $P=0.90$ & & $P=0.99$ & \\
\hline Normal & Ref & & Ref & & Ref & & Ref & \\
\hline Overweight & $1 \cdot 15$ & $1.00,1.31$ & 1.05 & $0.69,1.61$ & $1 \cdot 17$ & $1.02,1.34$ & 1.02 & $0.84,1.24$ \\
\hline Obese & 1.21 & $1.06,1.38$ & 1.33 & $0.86,2.06$ & $1 \cdot 11$ & $0.98,1.26$ & $1 \cdot 12$ & $0.87,1.45$ \\
\hline Maternal age (years) & & & & & $P=0.49$ & & $P=0.59$ & \\
\hline$<25$ & 0.96 & $0.79,1 \cdot 17$ & $1 \cdot 11$ & $0.52,2.36$ & $1 \cdot 11$ & $0.94,1.32$ & 1.07 & $0.78,1.46$ \\
\hline $25-29$ & 1.02 & $0.84,1.25$ & 0.91 & $0.55,1.51$ & 1.05 & $0.90,1.23$ & $1 \cdot 11$ & $0.79,1.56$ \\
\hline $30-34$ & 0.96 & $0.77,1.19$ & 0.70 & $0.43,1.16$ & $1 \cdot 11$ & $0.96,1.30$ & 1.05 & $0.76,1.44$ \\
\hline$\geq 35$ & Ref & & Ref & & Ref & & Ref & \\
\hline Parity & & & & & $P=0.02 \S$ & & $P=0.18 \S$ & \\
\hline 0 & Ref & & Ref & & Ref & & Ref & \\
\hline $1+$ & 0.89 & $0.78,1.00$ & 0.70 & $0.47,1.04$ & 1.05 & $0.94,1.19$ & 0.91 & $0.73,1.12$ \\
\hline Vitamin use\| & & & & & $P=0.93 \S$ & & $P=0.56 \S$ & \\
\hline Yes & 0.94 & $0.62,1.43$ & 1.20 & $0.79,1.84$ & 1.06 & $0.97,1.16$ & 0.99 & $0.82,1.20$ \\
\hline No & Ref & & Ref & & Ref & & Ref & \\
\hline Smoking during pregnancy & & & & & $P=0.23$ & & $P=0.15$ & \\
\hline Yes & 0.99 & $0.87,1.13$ & 1.41 & $0.85,2.33$ & 1.00 & $0.89,1.12$ & 0.71 & $0.36,1.42$ \\
\hline No & Ref & & Ref & & Ref & & Ref & \\
\hline Maternal education & & & & & $P=0.15$ & & $P=0.58$ & \\
\hline$<$ High school graduate & 1.30 & $1.03,1.65$ & 2.07 & $1.03,4.14$ & 0.93 & $0.78,1.11$ & $2 \cdot 62$ & $1 \cdot 10,6 \cdot 25$ \\
\hline High school graduate/GED & $1 \cdot 11$ & $0.89,1.39$ & 2.07 & $1.03,4.14$ & 0.90 & $0.77,1.05$ & $2 \cdot 50$ & $1.03,6.09$ \\
\hline Some college & 1.23 & $1.01,1.50$ & 1.40 & $0.76,2.57$ & 1.00 & $0.89,1.12$ & $2 \cdot 56$ & $0.99,6.59$ \\
\hline College graduate & Ref & & Ref & & Ref & & Ref & \\
\hline Marital status & & & & & $P=0.05 \S$ & & $P=0.05 \S$ & \\
\hline Never married & $1 \cdot 10$ & $0.94,1.29$ & 0.95 & $0.42,2 \cdot 15$ & 1.01 & $0.90,1.13$ & 1.27 & $0.99,1.62$ \\
\hline Married & Ref & & Ref & & Ref & & Ref & \\
\hline Living with partner & 1.00 & $0 \cdot 85,1 \cdot 18$ & 0.46 & $0.24,0.91$ & 0.90 & $0.77,1.06$ & $1 \cdot 10$ & $0.85,1.42$ \\
\hline Divorced/widowed/other & 1.00 & $0.78,1.24$ & 0.72 & $0.27,1.92$ & 0.97 & $0.80,1.18$ & 1.07 & $0.75,1.54$ \\
\hline Gestational age at enrolment & & & & & $P=0.42$ & & $P=0.46$ & \\
\hline 1st trimester & Ref & & Ref & & Ref & & Ref & \\
\hline 2nd trimester & 1.03 & $0.92,1.15$ & $1 \cdot 18$ & $0.80,1.73$ & 0.98 & $0.89,1.09$ & 0.98 & $0.82,1.16$ \\
\hline
\end{tabular}

Ref, reference; N/A, not applicable; GED, general education development.

* Insufficient vitamin D status is defined as plasma concentrations $<50 \mathrm{nmol} / \mathrm{l}$.

t $P$ values for effect-measure modification comparing non-Hispanic black with non-Hispanic white women.

$\ddagger P$ values for effect-measure modification comparing Hispanic with non-Hispanic white women.

$\S$ Statistically significant effect-measure modification $(P<0.10)$.

|| Includes any use of prenatal vitamin or multivitamins at time of enrolment.

for identifying women at high risk for vitamin D insufficiency during pregnancy. Findings from our study and others suggest that non-Hispanic black women are at higher risk of insufficiency and should be considered for targeted screenings and interventions. Moreover, while all women have some degree of seasonal variation in vitamin $\mathrm{D}$, low sunlight exposure in the winter may have a greater impact on insufficiency among non-Hispanic white women. This racial/ethnic difference in seasonal patterns could be useful for informing when and how frequently women should be screened throughout the year. Future research on how patterns and predictors of vitamin $\mathrm{D}$ differ by race/ethnicity will help inform targeted screening and interventions for insufficient vitamin D during pregnancy. Another important implication of these findings is that future studies of race/ethnicity differences in associations between prenatal vitamin $\mathrm{D}$ and health outcomes should address the potential for race/ethnicity-specific confounding using appropriate statistical approaches ${ }^{(14)}$.

Our results should be considered in the context of some limitations. Most notably, 25(OH)D concentrations were measured once during pregnancy. To assess individual-level seasonal patterns, future studies should measure $25(\mathrm{OH}) \mathrm{D}$ at multiple time points during pregnancy. While we used a $25(\mathrm{OH}) \mathrm{D}$ enzyme immunoassay that has been validated in pregnant women, it is no longer considered the 'gold-standard' measure of $25(\mathrm{OH}) \mathrm{D}$. However, validation studies have found concordance between immunoassays and the 'gold-standard' liquid chromatography measure of $25(\mathrm{OH}) \mathrm{D}^{(18)}$. Additionally, our enzyme immunoassay does not capture epimeric vitamin D metabolites, the biological relevance of which is currently 
debated in the field ${ }^{(19)}$. Because the vitamin D plasma collection and assay process was standardised within this study, we do not expect slight differences in $25(\mathrm{OH}) \mathrm{D}$ measurement to meaningfully change the interpretation of predictors and effect-measure modification by race/ethnicity in our paper. We also lacked data on important predictors of vitamin $\mathrm{D}$ such as amount of sun exposure and intake of vitamin D-rich foods. Future studies should replicate these results in geographically diverse study populations and using more detailed covariate data.

\section{Conclusions}

In this multi-ethnic population of pregnant women in southeastern USA, we confirmed that non-Hispanic black women are most at risk for vitamin D insufficiency. Additionally, we found evidence that certain predictors of vitamin D insufficiency during pregnancy - most notably, season - were statistically different by race/ethnicity with greater influence on insufficiency among non-Hispanic white women. Future studies identifying pregnancies at risk of vitamin $\mathrm{D}$ insufficiency should consider how predictors interact with race/ethnicity.

\section{Acknowledgements}

The authors would like to thank Rachel Maguire (data manager) and all study participants for their contributions to this research.

This work was supported by the NIEHS grant R01ES016772, NICHD T32 Reproductive, Perinatal, Pediatric Epidemiology Training Grant (T32HD052468), and the NIDDK R01DK085173/DK grant.

D. C., J. L. D. and J. P. B. conceived of the study objectives. C. H., B. F. F. and S. E. B. N. were involved in the original study design and data collection. D. C. and J. P. B. conducted the analyses. All authors contributed to the writing and development of the manuscript.

There were no conflicts of interest.

\section{References}

1. Bodnar LM, Simhan HN, Powers RW, et al. (2007) High prevalence of vitamin D insufficiency in black and white pregnant women residing in the northern United States and their neonates. J Nutr 137, 447-452.

2. Mulligan ML, Felton SK, Riek AE, et al. (2010) Implications of vitamin $\mathrm{D}$ deficiency in pregnancy and lactation. Am J Obstet Gynecol 202, 429.e1-429.e9.
3. Andersen LB, Jorgensen JS, Jensen TK, et al. (2015) Vitamin D insufficiency is associated with increased risk of first-trimester miscarriage in the Odense Child Cohort. Am J Clin Nutr 102, 633-638.

4. Bodnar LM, Simhan HN, Catov JM, et al. (2014) Maternal vitamin $\mathrm{D}$ status and the risk of mild and severe preeclampsia. Epidemiology 25, 207-214.

5. Bodnar LM, Catov JM, Zmuda JM, et al. (2010) Maternal serum 25-hydroxyvitamin D concentrations are associated with smallfor-gestational age births in white women. J Nutr 140, 999-1006.

6. Rosen CJ, Abrams SA, Aloia JF, et al. (2012) IOM committee members respond to Endocrine Society vitamin D guideline. J Clin Endocrinol Metab 97, 1146-1152.

7. Burris HH, Thomas A, Zera CA, et al. (2015) Prenatal vitamin use and vitamin $\mathrm{D}$ status during pregnancy, differences by race and overweight status. J Perinatol 35, 241-245.

8. Luque-Fernandez MA, Gelaye B, Vander Weele T, et al. (2014) Seasonal variation of 25-hydroxyvitamin-D among non-Hispanic black and white pregnant women from three US pregnancy cohorts. Paediatr Perinat Epidemiol 28, 166-176.

9. Tian Y, Holzman C, Siega-Riz AM, et al. (2016) Maternal serum 25-hydroxyvitamin D concentrations during pregnancy and infant birthweight for gestational age: a three-cohort study. Paediatr Perinat Epidemiol 30, 124-133.

10. Hoyo C, Murtha AP, Schildkraut JM, et al. (2011) Folic acid supplementation before and during pregnancy in the Newborn Epigenetics STudy (NEST). BMC Public Health 11, 46.

11. Ross AC, Taylor CL Yaktine AL, et al. (2011) Dietary Reference Intakes for Calcium and Vitamin D. Institute of Medicine (US) Committee to Review Dietary Reference Intakes for Vitamin D and Calcium. Washington, DC: National Academies Press.

12. Holick MF, Binkley NC, Bischoff-Ferrari HA, et al. (2011) Evaluation, treatment, and prevention of vitamin D deficiency: an Endocrine Society clinical practice guideline. I Clin Endocrinol Metab 96, 1911-1930.

13. Richardson DB, Kinlaw AC, MacLehose RF, et al. (2015) Standardized binomial models for risk or prevalence ratios and differences. Int J Epidemiol 44, 1660-1672.

14. Buckley JP, Doherty BT, Keil AP, et al. (2017) Statistical approaches for estimating sex-specific effects in endocrine disruptors research. Environ Health Perspect 125, 067013.

15. Libon F, Cavalier E \& Nikkels AF (2013) Skin color is relevant to vitamin D synthesis. Dermatology 227, 250-254.

16. Pereira-Santos M, Costa PR, Assis AM, et al. (2015) Obesity and vitamin $\mathrm{D}$ deficiency: a systematic review and meta-analysis. Obes Rev 16, 341-349.

17. Ozias MK, Kerling EH, Christifano DN, et al. (2014) Typical prenatal vitamin $\mathrm{D}$ supplement intake does not prevent decrease of plasma 25-hydroxyvitamin D at birth. I Am Coll Nutr 33, 394-399.

18. Roth HJ, Schmidt-Gayk H, Weber H, et al. (2008) Accuracy and clinical implications of seven 25 -hydroxyvitamin D methods compared with liquid chromatography-tandem mass spectrometry as a reference. Ann Clin Biochem 45, 153-159.

19. Benjamin Neelon SE, White AJ, Vidal AC, et al. (2018) Maternal vitamin D, DNA methylation at imprint regulatory regions and offspring weight at birth, 1 year and 3 years. Int J Obes (Lond) 42, 587-593. 